ECT*-97-002, CU-TP-814/97, DPNU-97-11, LUNFD6/(NFFL-7137)1997

\title{
Quantum Statistical Correlations and Single Particle Distributions for Slowly Expanding Systems with Temperature Profile
}

\author{
J. Helgesson ${ }^{1}$, T. Csörgö ${ }^{2,3}$, M. Asakawa ${ }^{4}$, and B. Lörstad ${ }^{5}$ * \\ ${ }^{1}$ ECT $^{*}$, European Centre for Studies in Theoretical Nuclear Physics \\ and Related Areas, Villa Tambosi, \\ Strada delle Tarabelle 286, I-38050 Villazzano (Trento), Italy \\ 2 MTA KFKI RMKI, H-1525 Budapest 114, P.O. Box 49, Hungary, \\ 3 Department of Physics, Columbia University, \\ 538 W 120th Street, New York, NY 10027, USA, \\ ${ }^{4}$ Department of Physics, School of Science, Nagoya University, \\ Nagoya, 464-01, Japan \\ 5 Department of Physics, University of Lund, \\ Box 118, S - 22100 Lund, Sweden
}

(February 11, 1997)

\begin{abstract}
Competition among particle evaporation, temperature gradient and flow is investigated in a phenomenological manner, based on a simultaneous analysis of quantum statistical correlations and momentum distributions for a non-relativistic, spherically symmetric, three-dimensionally expanding, finite source. The parameters of the model emission function are constrained by fits to neutron and proton momentum distributions and correlation functions in intermediate energy heavy-ion collisions. The temperature gradient is related to the momentum dependence of the radius parameters of the two-particle correlation function, as well as to the momentum-dependent temperature parameter of the single particle spectrum, while a long duration of particle evaporation is found to be responsible for the low relative momentum behavior of the two-particle correlations.
\end{abstract}

PACS: 25.70.-z

Typeset using REVTEX

*helgesso@ect.unitn.it, csorgo@rmki.kfki.hu, yuki@nuc-th.phys.nagoya-u.ac.jp, bengt@quark.lu.se 


\section{INTRODUCTION}

It has been shown recently that for non-relativistic, three-dimensionally expanding systems the quantum statistical correlations measure only part of the particle source and that the effective temperature of the momentum distribution is obtained as a combination of the freeze-out temperature and of the "geometrical temperature", a term due to expansion and to the finite geometrical sizes [1]. The expansion makes the effective radius parameters of the two-particle correlation functions smaller than the geometrical size of the source even for non-relativistic expanding systems [1].

Here we extend our study of non-relativistic expanding particle sources to the case of a temperature gradient inside the source. Such an extension of the model of Ref. [1] is motivated by data on momentum distribution of protons in heavy ion reactions in the nonrelativistic energy domain, which show deviations from the purely exponential spectrum [2]:3], although the neutron spectrum is well approximated by a Boltzmann distribution.

The purpose of our paper is to investigate the features of non-relativistic heavy ion collisions at a phenomenological level, parameterizing the emission function assuming local thermal equilibrium, without attempting to describe the microscopic mechanisms which lead to such a particle emission pattern. However, we include phenomenologically general features like evaporation, cooling, temperature gradient and flow as well as strong and Coulomb final state interactions for protons and neutrons, and test the resulting model on data at $E=30$ $\mathrm{MeV} /$ nucleon. Analytic approximations are also derived in order to gain improved insight into the influences of the competing effects. These analytic results are not directly applicable to nucleon correlations. However, they may be applicable to study pion correlation functions and spectra in such reactions where a spherical non-relativistic source may be assumed.

At high energies a relativistic analogue model [4 has been successful in describing the available data [5]. However there are important qualitative differences between relativistic heavy ion collisions at CERN SPS and those at non-relativistic energies from the point of view of particle sources. Low and intermediate energy reactions may create a very longlived, evaporative source, with characteristic lifetimes of a few $100 \mathrm{fm} / \mathrm{c}$, in contrast to the relatively short-lived systems of lifetimes not larger than a few $10 \mathrm{fm} / \mathrm{c}$ at CERN SPS. During such long evaporation times, cooling of the source is unavoidable and has to be included into the model, while the freeze-out at a constant temperature is a much better description at higher energies. Furthermore, in the non-relativistic heavy ion collisions mostly protons and neutrons are emitted and they have much stronger final state interactions than the pions dominating the final state at ultra-relativistic energies (for recent reviews on nucleon interferometry, see for example Refs. [6-8]).

We would like to emphasize that it is not our purpose to re-invent a detailed microscopical description of non-relativistic nuclear collisions. We are trying to develop a framework to describe the particle emission patterns, i.e., a simultaneous description of the invariant momentum distribution and the quantum statistical correlation function, from intermediate energies up to the highest energies achievable. In this paper we concentrate on the low part of the intermediate energy range. For example, sophisticated microscopical transport descriptions [9], such as the BUU (Boltzmann-Uehling-Uhlenbeck) and the QMD (Quantum Molecular Dynamics) models, are well-known and believed to provide a reasonable picture of proton emission in central heavy ion collisions from a few tenths up to hundreds of $\mathrm{MeV}$ per 
nucleon. However, in Ref. [10, the BUU model predicts too large correlations and underpredicts the number of protons emitted with low energies, for the reaction ${ }^{36} \mathrm{Ar}+{ }^{45} \mathrm{Sc}$ at $\mathrm{E}=120$ and $160 \mathrm{MeV} /$ nucleon. This indicates that the simultaneous description of twoparticle correlations and single-particle spectra is a rather difficult task. For energies below a few tens of $\mathrm{MeV}$ per nucleon, where long-lived evaporative particle emission is expected to dominate, the measured two-proton correlation functions were found to be consistent with compound-nucleus model predictions [11]; however, a simultaneous analysis of proton and neutron single particle spectra and two-particle correlation has not yet been performed to the best of our knowledge.

The basic model, including a temperature gradient, is presented in section IIA. Section IIB contains an extension of the model to long emission times. Analytic approximations for momentum distributions and correlation functions are derived in section III, and applied in section IV to situations where final state interactions can be ignored. Section $\square$ contains numerical applications to neutron and proton interferometry, where final state interactions are important. Finally, our results are summarized in section V1, while appendix A contains additional material on analytical approximations for the momentum distribution.

\section{THE MODEL}

The model presented in this section is based on the work of Ref. [四]. For clarity we briefly summarize the formalism along the lines of Ref. [1], together with a thorough description of the extensions.

\section{A. Emission Function with a Temperature Gradient}

The emission is characterized by the emission function or source function $S(x, p)$ which is the probability that a particle is produced at a given $x=(t, \boldsymbol{r})=\left(t, r_{x}, r_{y}, r_{z}\right)$ point in space-time with the four-momentum $p=(E, \boldsymbol{p})=\left(E, p_{x}, p_{y}, p_{z}\right)$, where the particle is on mass shell, $m^{2}=E^{2}-\boldsymbol{p}^{2}$. The quantum-mechanical analogy to the classical emission function is the time-derivative of the non-relativistic Wigner-function [12 which is analogous to the covariant Wigner-function of Ref. [13]. In this work the time-derivative of the Wignerfunction will be approximated by classical emission functions.

In terms of the emission function both the invariant momentum distribution (IMD) and the quantum statistical correlation function (QSCF) are prescribed. The Fouriertransformed emission function is introduced as an auxiliary quantity

$$
\tilde{S}(\Delta k ; K)=\int d^{4} x S(x ; K) \exp (i \Delta k \cdot x)
$$

where

$$
\Delta k=p_{1}-p_{2}, \quad K=\frac{p_{1}+p_{2}}{2}
$$

and $\Delta k \cdot x$ stands for the four-product of the four-vectors. Then the momentum distribution of number of the emitted particles, $N_{1}(\boldsymbol{p})$ is given by 


$$
N_{1}(\boldsymbol{p})=\frac{d^{3} n}{d p_{x} d p_{y} d p_{z}}=\tilde{S}(\Delta k=0, K=p),
$$

which is normalized to the mean multiplicity as

$$
\int d^{3} p N_{1}(\boldsymbol{p})=\langle n\rangle .
$$

Note that this normalization condition is different from the one used in Ref. [1] where the momentum distribution has been normalized to unity. We also assume that the nonrelativistic measure of the momentum space should be applied, due to the non-relativistic nature of the considered problem.

In the plane-wave approximation (i.e. neglecting final state interactions), the BoseEinstein or Fermi-Dirac correlation functions are prescribed in terms of our auxiliary function as

$$
C(K, \Delta k) \simeq 1 \pm \frac{|\tilde{S}(\Delta k, K)|^{2}}{|\tilde{S}(0, K)|^{2}},
$$

where the + sign stands for bosons and the - sign for fermions. This approximation involves an off-shell continuation of the on-shell emission functions, the significance of which was discussed first in Refs. 112, [14].

For central heavy ion collisions at intermediate energies the target and the projectile form a collective state which can be described as a non-relativistically expanding fluid within the framework of hydrodynamical models. Due to the expansion, the fluid cools and we assume that it then undergoes a certain disintegration process. In case the information about the initial directions is lost, the final freeze-out stage becomes approximately spherically symmetric.

We assume along the lines of Ref. [1] that the emission function is characterized by a distribution of production points $I(\boldsymbol{r})$ and by a distribution of the particle emission times, $H(t)$. The correlations between space-time and momentum-space shall be introduced by a non-relativistic momentum distribution. We assume that the expanding system is dilute enough when the particles are emitted so that the quantum statistical single-particle distribution can be well approximated by a Boltzmann distribution,

$$
\begin{aligned}
f(x ; p) & =\frac{g}{(2 \pi)^{3}} \exp \left(-\frac{(\boldsymbol{p}-m \boldsymbol{u}(x))^{2}}{2 m T(x)}\right) I(x), \\
I(x) & =\exp \left(\frac{\mu(x)}{T(x)}\right)
\end{aligned}
$$

Here $g$ is the degeneracy factor, $\boldsymbol{u}(x)$ is the (non-relativistic) flow velocity, the freeze-out temperature is denoted by $T$ and $\mu(x)$ is the chemical potential.

Thus the emission function is characterized as

$$
S(x ; K)=f(x ; K) H(t) .
$$

In order to simplify the results we shall keep only the mean and the width of the source distributions, i.e., we shall apply the Gaussian approximations for the distribution functions of $t$ and $\boldsymbol{r}$ as follows 


$$
\begin{aligned}
& I(x)=\exp \left(\frac{\mu_{0}}{T_{0}}\right) \exp \left(-\frac{\boldsymbol{r}^{2}}{2 R_{G}^{2}}\right), \\
& H(t)=\frac{1}{\left(2 \pi \Delta t^{2}\right)^{1 / 2}} \exp \left(-\frac{\left(t-t_{0}\right)^{2}}{2 \Delta t^{2}}\right) .
\end{aligned}
$$

(The expression in Eq. (10) is not suitable for very long emission times and evaporative processes. Such scenarios will be treated and discussed in the next section.)

In other words, we have the following ansatz for the chemical potential $\mu(x)$ :

$$
\frac{\mu(x)}{T(x)}=\frac{\mu_{0}}{T_{0}}-\frac{r^{2}}{2 R_{G}^{2}}
$$

which is analogous to the ones used in Refs. [1, 15].

We assume the following form of the local temperature distribution:

$$
T(\boldsymbol{r})=\frac{T_{0}}{1+a^{2} \boldsymbol{r}^{2} / 2 t_{0}^{2}} .
$$

The parameter $a$ controls the gradient of the local temperature at the last interaction point. We will in this work only treat the case when the temperature profile is decreasing as a function of $r$, though the expression (12) contains also increasing temperature profiles by taking a pure imaginary. Note, that the particular choice of the temperature profile is not influencing the leading order, approximate results: namely, we shall apply a saddle-point approximation and an expansion of the inverse temperature profile around the $\boldsymbol{r}=0, t=t_{0}$ point. Thus any other temperature profile, which leads to similar expansion coefficients, shall lead to similar approximate results.

We assume that the freeze-out temperature at each emission point is much smaller than the mass of the particles in this non-relativistic case,

$$
T(\boldsymbol{r}, t) \ll m .
$$

We select a velocity of the 3D expanding matter at space-time point $x$ so that it be spherically symmetric and describe an expansion in all three directions with a constant gradient. Thus the velocity around the mean freeze-out time $t_{0}$ is assumed to have the form

$$
\boldsymbol{u}(x)=b \frac{\boldsymbol{r}}{t_{0}}
$$

which describes a scaling solution of the non-relativistic hydrodynamical equations at the mean freeze-out time $t_{0}$ for $|\boldsymbol{r}| \ll t_{0}$ and $b=1$, Ref. [16]. Here we have introduced the parameter $b$ which controls the amount of flow. For $b=0$ we recover the case without flow. The results given in Ref. [1] are re-obtained for the case $a=0$ and $b=1$.

In Ref. [1] it was implicitly assumed that the duration of the particle emission is short, $\Delta t \ll t_{0}$, since the flow field, the geometrical radius and the freeze-out temperature were assumed not to change significantly during the time interval $\Delta t$, centered on $t_{0}$. The duration of the particle emission, $\Delta t$, has thus to satisfy

$$
\Delta t \ll \min \left(\frac{t_{0}}{a}, \frac{t_{0}}{b}\right)
$$


in order to warrant the model assumptions. If we have $a, b \ll 1$ then $\Delta t \approx t_{0}$ becomes possible since we approach the static fire-ball case.

When the above approximations for the emission function are valid, the auxiliary function can be rewritten as

$$
\tilde{S}(\Delta k, K)=\tilde{H}(\Delta E) \int d^{3} r \exp (-i \boldsymbol{k} \cdot \boldsymbol{r}) f\left(t_{0}, \boldsymbol{r} ; K\right)
$$

where $\tilde{H}(\Delta E)$ stands for the Fourier-transformed freeze-out time distribution. Within this approximation the freeze-out time distribution determines the energy-difference dependent part of the correlation function.

\section{B. Extension to Long Emission Times}

For low and intermediate energy nuclear reactions the duration time for the particle emission can be quite long. Evaporative models (with long duration times) have been fairly successful in describing certain aspects of such reactions [17, 18. The model presented above can be extended to also include long emission times by introducing cooling of the source and by modifying the time distribution.

The effect of possible cooling of the source can be caused, e.g., by the expansion and by the evaporation of the particles from the source. Although cooling can be consistently incorporated in the model of section II A (based on hydrodynamical results) it is very difficult to estimate analytically the effects for a long-lived, slowly cooling source, when particles are emitted during a large time interval. We therefore instead choose to utilize a phenomenological cooling profile, i.e. we assume that the decrease of the local temperature is given by

$$
T(\boldsymbol{r}, t)=T(\boldsymbol{r})\left(\frac{\tau}{t}\right)^{\alpha}
$$

where $T(\boldsymbol{r})$ is given by Eq. (12) and we use values $\tau=100 \mathrm{fm} / c$ and $\alpha=1 / 3$, motivated by a profile for isentropic three-dimensional expansion of and ideal gas. Note, that this form may not be a good approximation for the initial stage of the reaction since such a form cannot describe the initial rise of the temperature. However, we numerically found, that a long particle evaporation time is needed to get a simultaneous description of particle spectra and correlations in intermediate energy heavy ion collisions. Thus, most of the particles are emitted much later than the initial stage of the reaction and the approximate profile given by the above equation may be suitable to describe the fall of the temperature during most of the particle emission. A more detailed temperature profile could be obtained by a fit to the local temperature distribution of a microscopical simulation including collective effects (re-scattering) and particle evaporation.

The Gaussian time distribution in Eq. (10) does not give a good description for small times, $t \approx 0$, when $\Delta t$ is large, and thus has to be modified. Closely related to the Gaussian approximation is a distorted Gaussian distribution, which vanishes for very small times. We assume the form

$$
H_{\Gamma}(t)=2 t d \exp \left(-t^{2} d\right) \theta(t)
$$


which has the mean emission time

$$
\langle t\rangle=\frac{1}{2} \sqrt{\frac{\pi}{d}}
$$

with the variance

$$
\sigma^{2}(t)=\left\langle t^{2}\right\rangle-\langle t\rangle^{2}=\frac{1}{d}\left(1-\frac{\pi}{4}\right) .
$$

This form is motivated by the fact that there must be an initial rise in the particle emission followed by a long exponential tail. Such a distribution is for example the gamma distribution in $t^{2}$, which contains two parameters. However, for very broad time distributions, the mean and the variance are not very sensitive to independent tuning of these two parameters. Thus considering the current precision of the data (that is discussed in section $\bar{\nabla}$ ), we have fixed the small $t$ behavior of the $H_{\Gamma}(t)$ curve to a linear rise, decreasing the number of free parameters by one and simplifying the numerical integrations at the same time. Note that the analytic expressions above for $\langle t\rangle$ and $\sigma^{2}(t)$ are valid only when cooling is excluded. When cooling is included the mean emission time and the variance will be somewhat modified (see also the results and discussion in section ( $)$.

The source function $S(x, p)$ with the time distribution, $H_{\Gamma}(t)$ in Eq. (18), describes an expanding source with long duration of the particle emission, including cooling of the source, Eq. (17). It thus describes the gross features of particle evaporation, which is believed to take place at low collision energies. However, as an evaporative model, some features are only approximately treated. For example, quantities like flow velocity $(\boldsymbol{u})$ and geometrical source size $\left(R_{G}\right)$ are taken time independent, while in a more rigorous treatment also such quantities would vary with the time. Examples of more refined evaporative models can be found in Refs. [17.[19]. Here, however, we want to utilize a rather simple model, containing few parameters, applicable in a wide energy range (at high energies particle evaporation is negligible). We want a model that can describe nucleon emission as well as pion emission, and we want the model to be simple enough so that certain analytic results can be derived. Thus when the model is applied near its low energy limit (as in section $\nabla$ ), the extracted quantities like flow velocity and source radius, will reflect time averages.

\section{ANALYTIC APPROXIMATIONS}

The integral for the auxiliary function $\tilde{S}(\Delta k, K)$ in equation (16), can be evaluated with the help of the saddle-point method, which is described in details in Refs. 15,20.21. At the saddle-point, the partial derivatives of the emission function with regard to either $r_{x}, r_{y}$ or $r_{z}$ vanish simultaneously.

The saddle point, $\boldsymbol{r}_{s}$ is found by assuming that $\left|\boldsymbol{r}_{s}\right| / t_{0} \ll 1$. In this case one can expand the saddle point equations around $\boldsymbol{r}=0$ and one can solve the saddle point equations in a linearized problem. Note that for $a=0$ the method yields exact results because the nonlinearity of the saddle-point equations is related to the non-vanishing values of $a$. Since we utilize an expansion for $|\boldsymbol{r}| / t_{0} \ll 1$, all shapes of the temperature profile which lead to the same second order expansion for small distances as Eq. (12) lead to the same results.

With the help of the above approximations, we may rewrite the emission function as 


$$
S(x, p)=c_{g} \exp \left(-\frac{\left(\boldsymbol{p}-m \boldsymbol{u}\left(\boldsymbol{r}_{s}(\boldsymbol{p})\right)\right)^{2}}{2 m T\left(\boldsymbol{r}_{s}(\boldsymbol{p})\right)}-\frac{\boldsymbol{r}_{s}(\boldsymbol{p})^{2}}{2 R_{G}^{2}}-\frac{\left(\boldsymbol{r}-\boldsymbol{r}_{s}(\boldsymbol{p})\right)^{2}}{2 R_{*}^{2}}\right) H(t),
$$

with

$$
c_{g}=\frac{g}{(2 \pi)^{3}} \exp \left(\frac{\mu_{0}}{T_{0}}\right)
$$

The mean emission point coincides with the saddle-point $\boldsymbol{r}_{s}(\boldsymbol{p})$, being

$$
\boldsymbol{r}_{s}(\boldsymbol{p})=b t_{0} \frac{\boldsymbol{p}}{a^{2} E_{k}(\boldsymbol{p})+b^{2} m+t_{0}^{2} T_{0} / R_{G}^{2}},
$$

where the kinetic energy is denoted by $E_{k}(\boldsymbol{p})=\boldsymbol{p}^{2} /(2 \mathrm{~m})$. The following result is obtained for $R_{*}$ :

$$
\begin{aligned}
\frac{1}{R_{*}^{2}(\boldsymbol{p})} & =\frac{1}{R_{G}^{2}}+\frac{1}{R_{T}^{2}(\boldsymbol{p})}, \\
R_{T}^{2}(\boldsymbol{p}) & =t_{0}^{2} \frac{T_{0}}{a^{2} E_{k}(\boldsymbol{p})+b^{2} m} .
\end{aligned}
$$

Within the above approximations, the auxiliary function contains a momentum-dependent factor which shall enter the momentum distribution only and another factor which is a Fourier-transform of a Gaussian and so easily integrable. The approximations are selfconsistent if the condition $\left|\boldsymbol{r}_{s}\right| / t_{0} \ll 1$ is satisfied. We return to this point later.

The analytic results for the momentum distribution and the quantum statistical correlation function are given as

$$
\begin{aligned}
N_{1}(\boldsymbol{p}) & =c_{g}\left(2 \pi R_{*}^{2}(\boldsymbol{p})\right)^{(3 / 2)} \exp \left(-\frac{\left(\boldsymbol{p}-m \boldsymbol{u}\left(\boldsymbol{r}_{s}(\boldsymbol{p})\right)\right)^{2}}{2 m T\left(\boldsymbol{r}_{s}(\boldsymbol{p})\right)}-\frac{\boldsymbol{r}_{s}(\boldsymbol{p})^{2}}{2 R_{G}^{2}}\right), \\
C(K, \Delta k) & =1 \pm \exp \left(-R_{*}^{2}(\boldsymbol{K}) \boldsymbol{\Delta} \boldsymbol{k}^{2}-\Delta t^{2} \Delta E^{2}\right) .
\end{aligned}
$$

The effects of final state Coulomb and Yukawa interactions on the two-particle relative wave-functions are neglected (in section $\nabla$ final state interactions are taken into account).

These expressions are generalizations of the momentum distribution and correlation function obtained in Ref. [1] and reduce to those for $a=0$ and $b=1$. The static fire-ball corresponds to the case $a=b=0$. A new feature for $a \neq 0$ is that the radius parameter of the QSCF becomes a decreasing function of the momentum and also the effective temperature becomes momentum dependent.

Similarly to the $a=0$ and $b=1$ case [1], the effective temperature $T_{*}$ shall be determined by the maximum of the local temperature, $T_{0}$, and the geometrical temperature defined as

$$
T_{G}=T_{0} \frac{R_{G}^{2}}{R_{T}^{2}}
$$

The relationship is given by

$$
\begin{aligned}
\frac{1}{T_{*}} & =\frac{f}{T_{0}+T_{G}}+\frac{1-f}{T_{0}}, \\
f & =\frac{b^{2}}{a^{2}+b^{2}}
\end{aligned}
$$




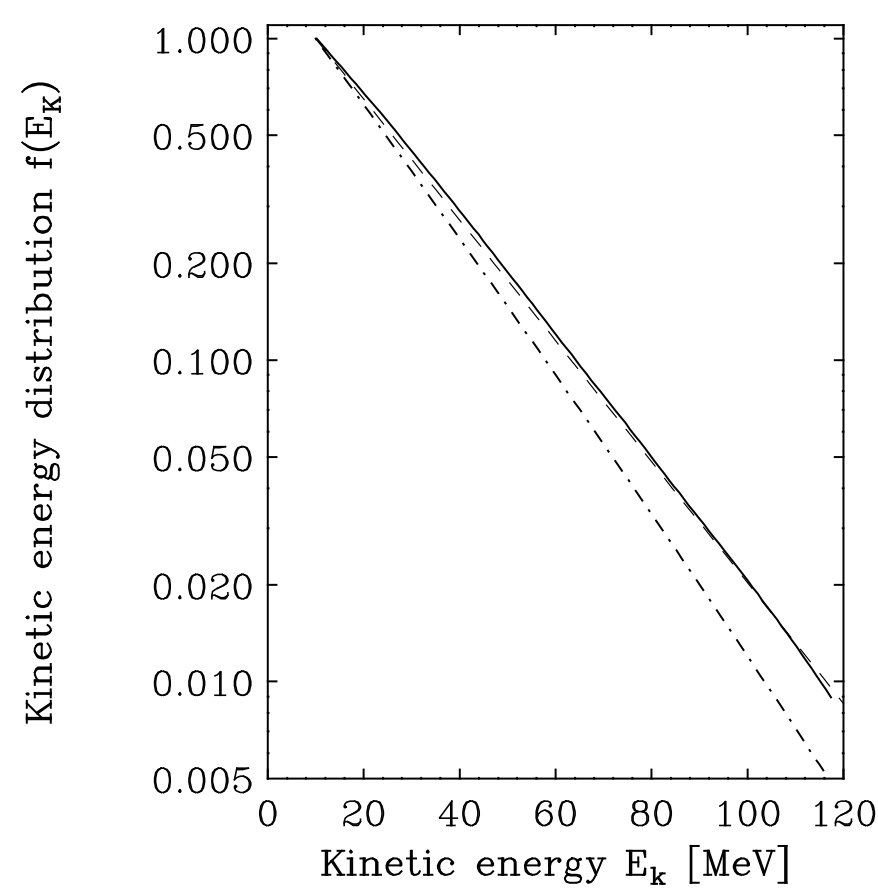

FIG. 1. Comparison of the numerically integrated energy distribution of protons (solid line) with a Boltzmann distribution of $T_{*}=23.1 \mathrm{MeV}$ (dashed line) and with analytical results for the linearized saddle-point calculation, Eq. (26) as indicated by the dash-dotted line, for the parameter values of $m=938.3 \mathrm{MeV} / c^{2}, R_{G}=7.6 \mathrm{fm}, T_{0}=10.0 \mathrm{MeV}, t_{0}=45.0 \mathrm{fm} / \mathrm{c}, a=1.0$, and $b=0.7$. The energy distributions, $f\left(E_{k}\right) \propto N_{1}(\boldsymbol{p}) / \boldsymbol{p}^{2}$, are re-scaled so that $f\left(E_{k, 0}\right)=1$ for $E_{k, 0}=10 \mathrm{MeV}$. Note the characteristic bending down of both the solid and the dash-dotted line as compared to the Boltzmann distribution.

which is analogous to the case obtained for the slope of the momentum distribution at high energies [4,24]. In the case of no temperature gradient, $T_{*}$ will grow linearly with the particle mass. In case of no flow, $T_{*}$ is independent of mass. For a given mass, the effective temperature $T_{*}$ shall be constant only in a limited $p$ interval, which is given by $p^{2} \ll m^{2} b^{2} / a^{2}$. Since the present investigation is limited to the non-relativistic $p \ll m$ region, it follows that the effective temperature is not noticeably momentum-dependent for small temperature gradient satisfying $a^{2} \leq b^{2}$. As soon as the temperature gradient increases above the $a^{2} \leq b^{2}$ region, a characteristic high momentum suppression may appear in the tail of the distribution, see Fig. 1 for example.

When fitting the model to data, we concentrate on the $a^{2} \geq 0$ case, since this is the case which may result in a suppression at high momentum - a phenomenon observed in the momentum distribution of protons in intermediate energy heavy ion reactions [2, 3].

A short discussion on the validity of the saddle-point approximation is presented in appendix A, together with suggested improvements of the approximation and alternative approximation schemes. 


\section{CORRELATIONS WITHOUT FINAL STATE INTERACTIONS}

In this section the analytic results will be applied to momentum distributions and correlation functions where final state interactions can be neglected. Applications could for example be pion distributions and correlations at BEVALAC energies, though the discussion in this section is quite general and could be applied also to other situations where final state interactions can be ignored. The qualitative discussion in this section also serves as a preparation to section $\nabla$, where final state interaction is introduced as well. The presentation will follow the lines of Ref. [1], since the results are very similar.

The relative momentum, $\boldsymbol{\Delta} \boldsymbol{k}$, which appears in the correlation function, is invariant under Galilei-transformations, but the energy difference is not invariant even under the nonrelativistic Galilei transformations. This can be re-formulated so that the specific directional dependence becomes more transparent. The energy difference is

$$
\Delta E=\frac{\boldsymbol{p}_{1}^{2}-\boldsymbol{p}_{2}^{2}}{2 m}=\left(\frac{\boldsymbol{p}_{1}+\boldsymbol{p}_{2}}{2}\right) \cdot \frac{\boldsymbol{\Delta} \boldsymbol{k}}{m}=\frac{\boldsymbol{K} \cdot \boldsymbol{\Delta} \boldsymbol{k}}{m}=\boldsymbol{v}_{\boldsymbol{K}} \cdot \boldsymbol{\Delta} \boldsymbol{k},
$$

where we have introduced the mean velocity of the pair, $\boldsymbol{v}_{\boldsymbol{K}}=\boldsymbol{K} / m$.

Let us define the out direction to be parallel to the mean velocity of the pair, $\boldsymbol{v}_{\boldsymbol{K}}$, and the perp index for the remaining two principal directions, both being perpendicular to the out direction. This naming convention corresponds to the one used in high energy heavy ion collisions [22,23].

By this definition, the principal directions, which will be utilized to evaluate the correlation function, are assigned to the given mean momentum of a given particle pair and not that of the fire-ball: by changing the mean momentum we change the out and the perp directions as well. In any given frame, the relative momentum can be decomposed as

$$
\Delta k=Q_{o u t}+Q_{\text {perp }}
$$

and the correlation function can be rewritten as

$$
\begin{aligned}
C\left(\boldsymbol{Q}_{\text {out }}, \boldsymbol{Q}_{\text {perp }}\right) & =1 \pm \exp \left(-R_{\text {perp }}^{2}(\boldsymbol{K}) \boldsymbol{Q}_{\text {perp }}^{2}-R_{\text {out }}^{2}(\boldsymbol{K}) \boldsymbol{Q}_{\text {out }}^{2}\right) \\
R_{\text {perp }}^{2}(\boldsymbol{K}) & =R_{*}^{2}(\boldsymbol{K})=\frac{R_{T}^{2}(\boldsymbol{K}) R_{G}^{2}}{R_{T}^{2}(\boldsymbol{K})+R_{G}^{2}} \\
R_{\text {out }}^{2}(\boldsymbol{K}) & =R_{*}^{2}(\boldsymbol{K})+\boldsymbol{v}_{\boldsymbol{K}}^{2} \Delta t^{2}
\end{aligned}
$$

Thus the perpendicular components shall measure the effective radius $R_{*}(\boldsymbol{K})$. What is the interpretation of this quantity?

There are two length-scales in the problem: the geometrical length-scale $R_{G}$ and the thermal one $R_{T}$, the latter being generated by the flow gradient $b / t_{0}$, the central temperature $T_{0}$ and the temperature gradient $a / t_{0}$. From the previous equation one can see that the effective $R_{*}(\boldsymbol{K})$, measured by the perp component of the correlation function, is dominated by the smaller of the two. In other words, for large and relatively cold three-dimensionally expanding systems, not the whole source can be seen by quantum statistical correlations, but only a part of the whole system, which is characterized by a thermal length-scale $R_{T}(\boldsymbol{K})$. Note also that, unlike in the case discussed in Ref. [1], the radius parameter $R_{*}(\boldsymbol{K})$ shall 


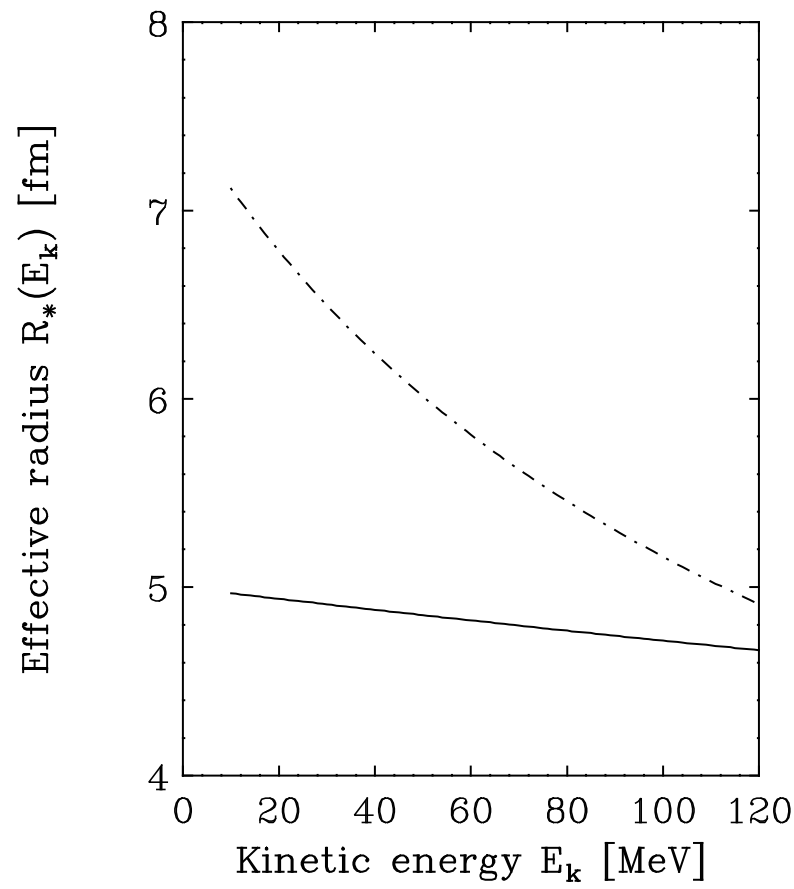

FIG. 2. Kinetic energy dependence of the effective radius parameter $R_{*}\left(E_{k}\right)$, Eq. 24-25). Solid line indicates $R_{*}\left(E_{k}\right)$ for the parameter values of Fig. 1, dash-dotted line stands for $a=2.0$, $b=0.1$, other parameters being the same as for the solid line.

not be a constant but it will depend on the momentum of the particles. See Fig. 2 for illustration.

From Eq. (35) it follows that the out component in general shall also be sensitive to the duration of the freeze-out time distribution, since it contains a term $\boldsymbol{v}_{\boldsymbol{K}}^{2} \Delta t^{2}$. This term vanishes in the center of mass frame (c.m.f.) of the particle pair, since $\Delta E=\left|\boldsymbol{v}_{\boldsymbol{K}}\right|=0$ in c.m.f. In this specific system,

$$
Q_{I}^{2}=\boldsymbol{Q}_{\text {perp }}^{2}+\boldsymbol{Q}_{\text {out }}^{2}-\Delta E^{2}=\boldsymbol{Q}_{\text {perp }}^{2}+\boldsymbol{Q}_{\text {out }}^{2} \quad \text { in c.m.f. of the pair }
$$

is the invariant momentum difference. The correlation function in the considered case becomes symmetric, when evaluated in the c.m. of the pair:

$$
C\left(Q_{I}\right)=1 \pm \exp \left(-R_{*}^{2}(\boldsymbol{K}) Q_{I}^{2}\right) \quad \text { in c.m.f. of the pair. }
$$

It is interesting to investigate another limiting case, $R_{G} \ll R_{T}(\boldsymbol{K})$. In this case we re-obtain the standard results:

$$
R_{\text {perp }}^{2}=R_{G}^{2}, \quad R_{\text {out }}^{2}=R_{G}^{2}+\boldsymbol{v}_{\boldsymbol{K}}^{2} \Delta t^{2}, \quad T_{*}=T,
$$

i.e., if the thermal length scale is larger than the geometrical size in all three directions, the correlation measurement determines the geometrical sizes properly, and the momentum distribution will be determined by the freeze-out temperature being just a thermal distribution for a static source. In this limiting case, the effective radius parameter as well as the effective temperature become independent of the momentum of the emitted particles.

The non-relativistic model presented in this work has six input parameters: $R_{G}, t_{0}, \Delta t$, $T, a$ and $b$, which determine two measurable functions. This, in turn, implies that all these 
parameters can in principle be determined from the detailed analysis of the momentum dependence of the two-particle correlation functions and from the deviation of the oneparticle invariant momentum distributions from the purely thermal behavior.

For the special case when the measured QSCF-s are found to be momentum-independent and the IMD is well described by a Boltzmann momentum distribution, the $a=0$ case is concluded. In this case, we may measure the parameters $\Delta t, R_{*}$ and $T_{*}$ which are constants. The measurable parameters $R_{*}$ and $T_{*}$ are determined by four model parameters, $R_{G}, T_{0}$, $t_{0}$ and $b$. Thus the model parameters cannot be determined uniquely from the IMD and QSCF observables in the $a=0$ special case. Inequalities can be obtained, along the lines of Ref. [1], to restrict the values of the model parameters in this special case. For example, we have $T_{0} \leq T_{*}$ and $R_{G} \geq R_{*}$. Note that a similar degeneracy of $T_{*}$ and $R_{*}$ has been found in Ref. [1] for the $a=0$ and $b=1$ special case, where it was observed that the the same spectra and correlations can be obtained for different values of the three input parameters.

The general results for the correlation function given in Eqs. (33-35) indicate structural similarity with the Bose-Einstein correlation function parameters for a class of models which includes relativistic longitudinal flows, non-relativistic transverse flows and a transverse temperature profile [4, 24]. The radius parameters of the correlation function are momentum dependent both for the non-relativistic model presented here and for the model-class discussed in Refs. [4,24]. The structural similarity implies that the effective duration parameter $\Delta t_{*}^{2}$, defined as the coefficient of $\boldsymbol{v}_{\boldsymbol{K}}^{2}$ in Eq. (35), shall become momentum dependent: $\Delta t_{*}=\Delta t_{*}(\boldsymbol{K})$ if the cooling effects are switched on. This new, momentum-dependent duration parameter $\Delta t_{*}(\boldsymbol{K})$ shall replace the momentum-independent $\Delta t$ duration parameter in the correlation function if the temporal changes of the temperature are significant.

\section{APPLICATION TO NEUTRON AND PROTON INTERFEROMETRY}

In section $\llbracket$ IV it was discussed that the effective radius parameter $R_{*}(\boldsymbol{K})$ is not constant but depends on the momentum of the particles. Such an effect has indeed been seen in the measured proton-proton correlation functions in the ${ }^{27} \mathrm{Al}\left({ }^{14} \mathrm{~N}, p p\right)$ reactions at $E=75$ $\mathrm{MeV} /$ nucleon [7,25]: the larger the momentum of the protons the smaller the effective source size [7]. This feature is in qualitative agreement with the analytic results given in section III], since the thermal radius $R_{T}(\boldsymbol{K})$ is a decreasing function of the absolute value of the mean momentum, $|\boldsymbol{K}|$. The effective source size is dominated by the smaller of the thermal and geometrical radius, thus it is also a decreasing function of the mean momentum of the particle pair.

The results presented in the previous sections contain the essential ingredients which are needed to obtain a momentum-dependent radius parameter in the non-relativistic domain. However, to be utilized for nucleons emitted in intermediate energy heavy ion reactions [6.7], also two other important effects need to be considered, namely cooling of the source (see section [IB) and final state interactions. Final state interactions can be included for $p p$ pairs utilizing the Coulomb + strong interactions and Fermi-Dirac statistics, the strong interactions and Fermi-Dirac statistics for $n n$ pairs and the strong interactions only for the $n p$ pairs, utilizing the Wigner-function formalism developed by S. Pratt and collaborators (see e.g. Refs. [7, 12 for the description of the method). Essentially, this calculation includes the evaluation of the two-particle relative wave-function with the above Coulomb and/or strong 
interaction using the appropriate quantum statistical (anti)symmetrization and averaging the result over the particle distribution calculated from the model in section IIA. A Reid soft core potential is used to take into account the strong final state interactions. The essential consequences of the final-state interactions are that they completely modify the short-range parts of the two-particle correlations, and they create a peak at low relative momentum in the correlation functions. In the case of proton pairs, this peak is suppressed by the Coulomb repulsion which creates a hole centered at zero relative momentum in the $p p$ correlation function.

Light particle interferometry at intermediate energy heavy-ion collisions has been extensively studied, both experimentally and theoretically. Many attempts, using different models have been put forward, for example: simple Gaussian source parameterizations, evaporation, and transport models such as QMD and BUU (see Refs. [7,8] and references therein). These models, containing different physical ingredients and information, have to different degrees been successful in describing the data.

One of the purposes of this paper is to test if the model, presented in section 【1, is applicable to $n n$ and $p p$ interferometry at intermediate energies. The model contains a rather small set of parameters, and by making simultaneous fits to $n$ and $p$ single energy spectra, and $n n$ and $p p$ correlation functions, rather hard constraints are put on the parameter set. Thus a qualitative and quantitative understanding of certain aspects of the source can be extracted.

The evolution of the particle emission in a heavy-ion collision at intermediate energies may roughly be described as: production of pre-equilibrium particles; expansion and possible freeze-out of a compound source; possible evaporation from an excited residue of the source. Note though, that this separation is not very distinct and there is an overlap between the different stages. The importance of the various stages above also depends on the beam energy and the impact parameter of the collision. The model presented in this paper describes well the second stage above and, for long emission times, also part of the third stage. If these stages give the main contribution to the emission of nucleons, a satisfactory description of experimental data can be obtained.

\begin{tabular}{|l|r|r|r|r|r|}
\hline \hline & $R_{G}(\mathrm{fm})$ & $T_{0}(\mathrm{MeV})$ & $a / t_{0}(\mathrm{fm} / c)^{-1}$ & $b / t_{0}(\mathrm{fm} / c)^{-1}$ & $d(\mathrm{fm} / c)^{-2}$ \\
\hline \hline Neutrons & 4.0 & 3.0 & 0.0 & 0.018 & $5.0 \cdot 10^{-5}$ \\
\hline Protons & 4.0 & 5.0 & 0.14 & 0.036 & $5.0 \cdot 10^{-5}$ \\
\hline \hline
\end{tabular}

TABLE I. Parameter values used for calculating $n$ and $p$ spectra and correlation functions

We have applied our model to the reaction ${ }^{40} \mathrm{Ar}+{ }^{197} \mathrm{Au}$ at $30 \mathrm{MeV} /$ nucleon, to compare with experimental single spectra and correlation functions from the $n p$ correlation experiments described in Refs. [3, 26, 27]. With the parameter set presented in table [ 1 we have obtained a simultaneous fit to $n$ and $p$ single spectra, as well as $n n$ and $p p$ correlation functions. Thus we have used the same parameters for neutrons as for protons, except for

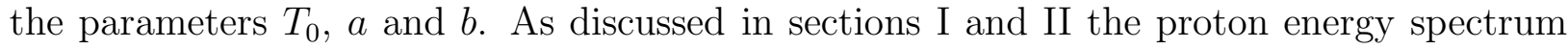
deviates from the thermal spectrum [2,3], although the neutron spectrum is well approximated by a Boltzmann distribution. The Coulomb interaction makes a difference between protons and neutrons, and this behavior is effectively obtained within our framework for protons by allowing a temperature gradient inside the source and a different flow parameter. 
Protons emitted from the middle of the source roll down from a higher Coulomb-potential than those from the surface, thus protons from the middle are emitted with a higher kinetic energy than those emitted from the surface. This qualitative feature is similar to a system which is hotter in the middle than at the surface. Incorporating a temperature gradient inside the source does effectively describe such an effect.

To keep the description as simple as possible in this first attempt to test the applicability of the model at intermediate energies, we have ignored impact parameter averaging and Coulomb interaction with the source. An averaging over different impact parameters could for example be done by allowing the radius parameter, $R_{G}$, to vary with the geometrical overlap of the projectile and target. However, such a prescription also introduces an additional uncertainty, and has therefore been ignored to keep the description simple. Thus the extracted source size will reflect a "mean" source size (assumed to be averaged over the different impact parameters).

When performing the calculations we have taken into account the experimental energy thresholds and the acceptance region of the experimental set up.

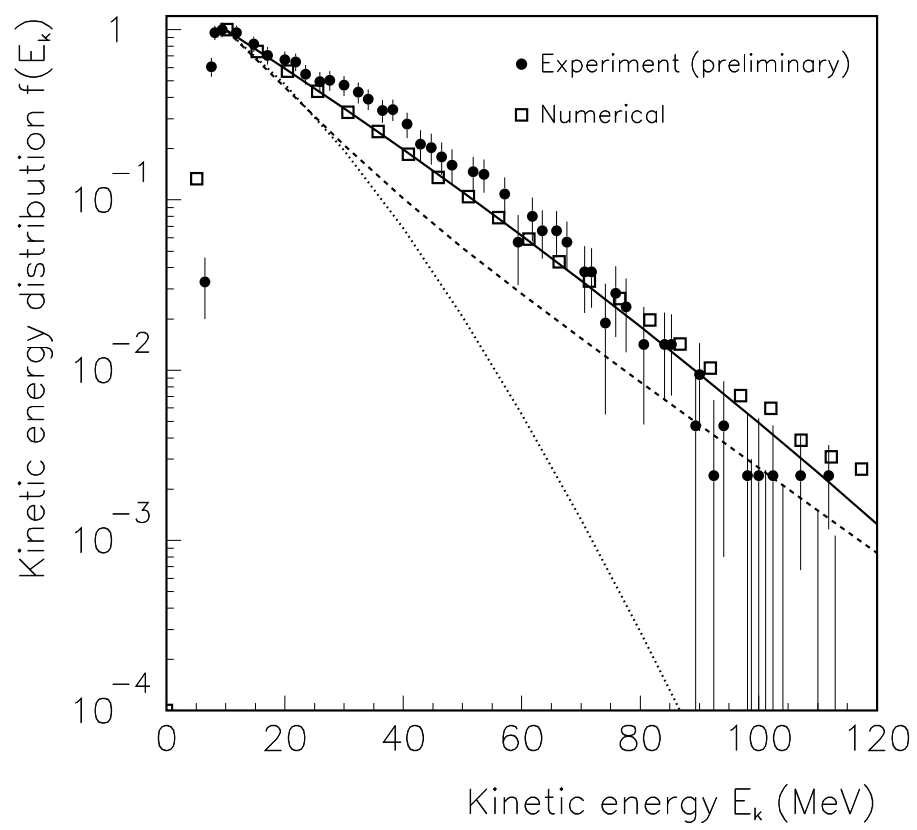

FIG. 3. Experimental and numerically calculated proton energy spectrum. The filled circles represent preliminary inclusive proton emission data [3] from the $n p$ correlation experiment described in Ref. [26], the open squares the full numerical simulation, while analytic approximations are represented by a solid curve (Eq. (A2)), dashed curve (Eq. (A1)) and a dotted curve (Eq. (26)), respectively. The experimental data are preliminary, without fully estimated errors, why we have estimated the errors from the fluctuations of the data points. The parameter set in table [1] was employed for the numerical calculations. The energy distributions, $f\left(E_{k}\right)$ are re-scaled so that $f\left(E_{k, 0}\right)=1$ for $E_{k, 0}=10 \mathrm{MeV}$.

Experimental inclusive proton emission data [3] (from the $n p$ correlation experiment described in Ref. [26]) are presented in Fig. 30 (filled circles) together with our fit (open squares). In addition we show the analytic approximate expression in Eqs. (26), (A1) and 
(A2) as a dotted, dashed and solid curve respectively. The neutron spectrum, not shown, is purely thermal with an effective temperature of $8.5 \pm 1 \mathrm{MeV}$ and is also well reproduced by our calculations.

Proton correlation functions are presented in Fig. 1 as a function of the relative momentum $q=\left|\boldsymbol{p}_{1}-\boldsymbol{p}_{1}\right| / 2$, integrated over the total pair momentum $\left(\boldsymbol{p}_{1}+\boldsymbol{p}_{2}\right)$. The experimental results are reproduced qualitatively, in some regions of the momentum space even quantitatively. Considering an experimental uncertainty in $q$ of $3.0 \leq \Delta q \leq 5.5 \mathrm{MeV} / c$ [27], which is not taken into account in our calculations, and the known difficulty to describe both the single-particle spectrum and the correlations for protons and neutrons at the same time [10], we think that our result is interesting. The discrepancy for $q<10-15 \mathrm{MeV} / c$ is partly due to the experimental uncertainty $\Delta q$. However, a discrepancy has also been seen in other models (see e.g. Ref. [8]) and suggested explanations are the following: more than one source, Coulomb interaction with the source or pre-equilibrium emission.

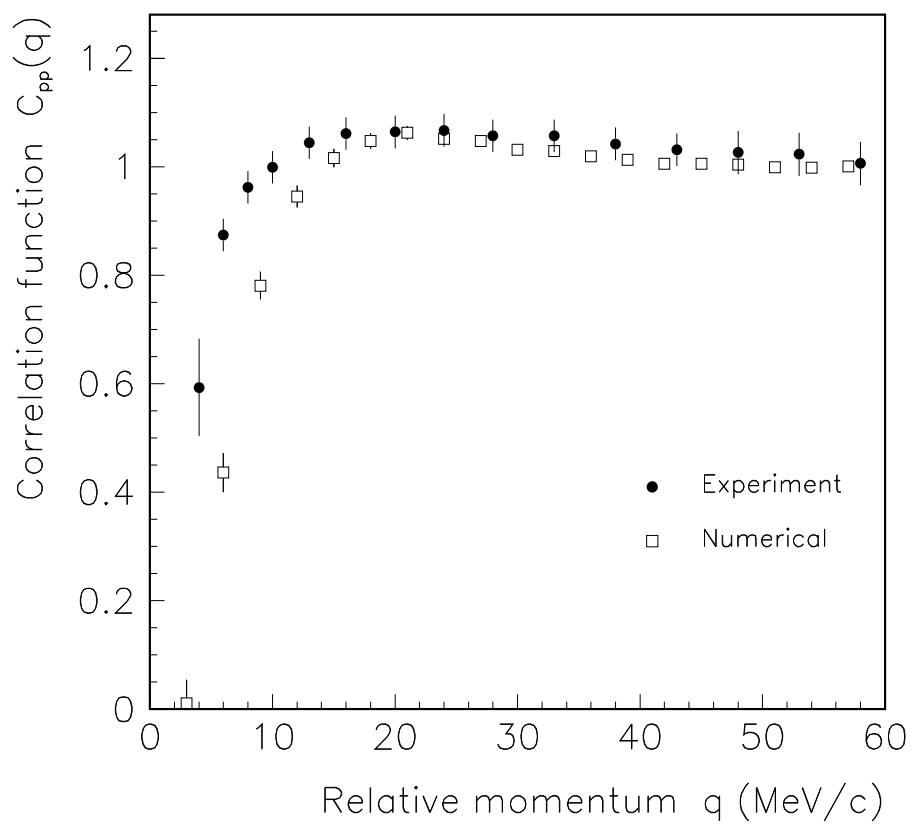

FIG. 4. Experimental and numerically calculated $p p$ correlation function. The filled circles represent data from the $n p$ correlation experiment described in Ref. [26], the open squares the full numerical simulation. The parameter set in table 1 was employed for the numerical calculations.

The experimental $n n$ correlation function contains some uncertainties, namely cross talk between the detectors, and experimental cuts at low relative momentum. From simulations, the cross talk (see Fig. 3e of Ref. [26] ) has been found to mainly contribute to the "bump" around $15<q<40 \mathrm{MeV} / c$. Furthermore the sharp rise in $C(q)$ at $q \approx 10 \mathrm{MeV} / c$ is based on rather few events with neutron kinetic energy smaller than about $10 \mathrm{MeV}$ (see Fig. $3 \mathrm{~d}$ of Ref. [26]) close to detector energy threshold and acceptance limits. The momentum uncertainty, $\Delta q$, is smaller than $2 \mathrm{MeV} / c$. Considering these uncertainties the experimental $C(q)$ is reproduced in an acceptable manner in Fig. 5 .

When performing the parameter fit we have found that it is not possible to simultaneously reproduce $n$ and $p$ energy spectra, as well as $n n$ and $p p$ correlation functions, unless 


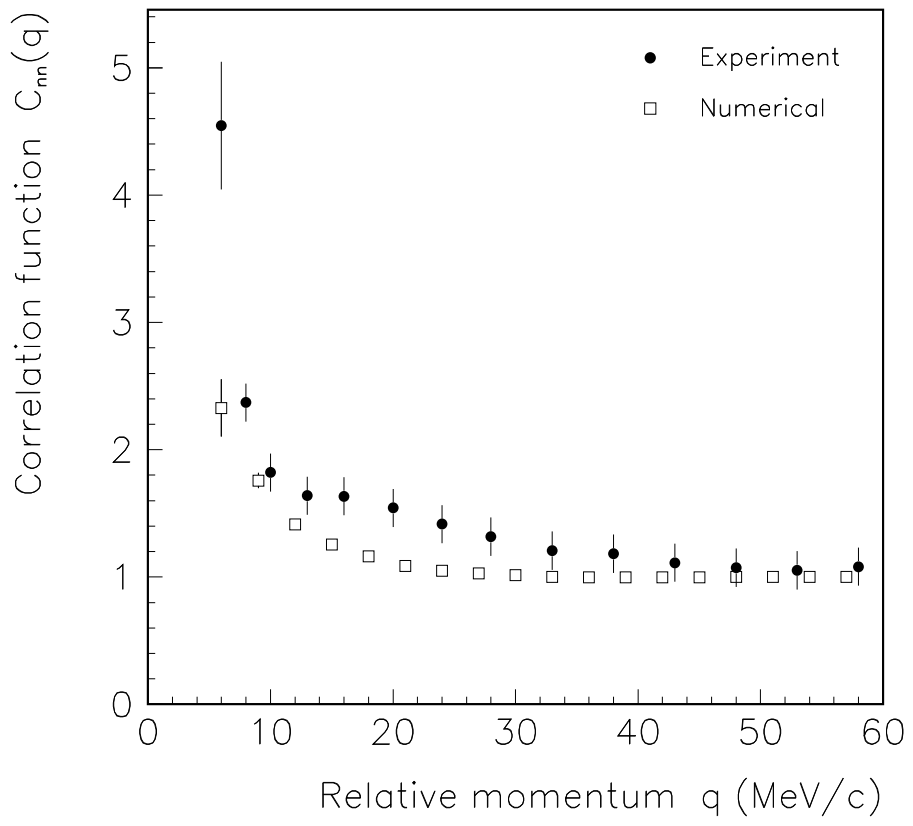

FIG. 5. Experimental and numerically calculated $n n$ correlation function. The filled circles represent data from the $n p$ correlation experiment described in Ref. [26], the open squares the full numerical simulation. The parameter set in table 1 was employed for the numerical calculations.

the duration of the particle emission is taken large (several hundreds of $\mathrm{fm} / \mathrm{c}$ ). The long duration is found to be responsible for the low relative momentum behavior of the twoparticle correlations. Thus we have used $H_{\Gamma}(t)$ in Eq. (18) for the time distribution. With the parameter set in table $\square$ we obtain numerically the mean emission time $\langle t\rangle$ and duration $\sigma(t)$ :

$$
\langle t\rangle \approx 520 \mathrm{fm} / c \quad \sigma(t) \approx 320 \mathrm{fm} / c
$$

(Note that $\langle t\rangle$ necessarily becomes large because of the broad time distribution limited to $t>0$.) This scenario is in agreement with the general view of nuclear reactions in the energy domain of a few tenths of $\mathrm{MeV} /$ nucleon, namely a rather long-lived excited source emitting particles. Note though that a pure evaporative source gives a worse description of the experimental $n n$ and $p p$ correlation functions (see Ref. [26]) and fails to reproduce the single particle energy distribution. Thus the inclusion of the expansion seems to be mandatory, although the extracted flow velocities are small.

The extracted geometrical source radius $R_{G} \approx 4 \mathrm{fm}$ is quite reasonable considering that a Gaussian parameterization is used (instead of a Woods-Saxon distribution, usually employed for the ground state density distribution). Note also that the extracted source size reflects an average over different times and impact parameters. The effective radius, $R_{*}$, seen in the correlation function is smaller than $R_{G} ; 1.1-1.8 \mathrm{fm}$ for protons (depending on momentum) and $2.5 \mathrm{fm}$ for neutrons.

From the experimental energy spectra we know that the effective temperature for neutrons should be $8.5 \pm 1 \mathrm{MeV}$ and for protons in the range $15-25 \mathrm{MeV}$ depending on the

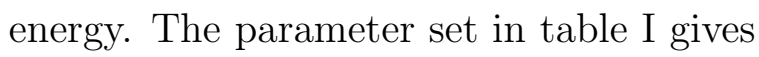




$$
T_{\mathrm{eff}}=T_{*} \approx 7.9 \mathrm{MeV}
$$

for neutrons, and for protons, using the Taylor approximation in Eq. (A2), we obtain

$$
T_{\text {eff }} \approx 20.1 \mathrm{MeV}, \quad E_{k}=10 \mathrm{MeV} ; \quad T_{\text {eff }} \approx 17.4 \mathrm{MeV}, \quad E_{k}=100 \mathrm{MeV}
$$

This is obtained with an input temperature of $T_{0}=3 \mathrm{MeV}$ for neutrons, and for protons with a temperature profile that decreases from $5 \mathrm{MeV}$ at the center of the source to $4.3 \mathrm{MeV}$ at $r=R_{G}$ and $3.1 \mathrm{MeV}$ at $r=2 R_{G}$.

The parameter set also gives the flow velocities

$$
u(n) \approx 0.018 r c ; \quad u(p) \approx 0.036 r c
$$

The results presented in Figs. 3 - 5 are obtained with cooling included according to Eq. 17. We have numerically found that the results change only to a minor extent if the cooling is excluded. Thus the different approximations in Eqs. (26), (A1) and (A2) are useful also when cooling is included.

A little note should be made on the parameter fit in our model. It is not possible to determine the parameters uniquely from the experimental data, though they are strongly constrained. Further constraints could be imposed by also investigating the dependence of the correlation function on the total pair momentum, though the current data set does not allow such an investigation.

\section{SUMMARY}

In summary, we have calculated the invariant momentum distribution and the quantum statistical correlation function in a non-relativistic model for spherically symmetric non-relativistic expansion with possible temperature gradient, phenomenologically including cooling and particle evaporation. We have thus presented a generalization of the previous work [1] by introducing new parameters. These parameters control the strength of the flow, the temperature gradient, the strength of cooling and the duration of particle emission.

We have observed structural similarity of this non-relativistic model to the relativistic expansion described in Refs. [4,24], namely the effective radius parameters of the two-particle correlation functions and the effective slope parameters of the single particle spectra became momentum dependent due to the interplay of the local thermal scales and the geometrical scales. On this level, the model presented here is very similar to the relativistic one.

The main effects of the temperature gradient are that it introduces i) a momentumdependent effective temperature which is decreasing for increasing momentum, resulting in a suppression at high momentum as compared to the Boltzmann distribution; ii) a momentumdependent effective source size which decrease with increasing total momentum. These qualitative features have been seen in non-relativistic heavy-ion collisions.

The model presented here, including final state interactions, has been applied to measured correlation functions and preliminary neutron and proton energy spectra in the reaction ${ }^{40} \mathrm{Ar}+{ }^{197} \mathrm{Au}$ at $30 \mathrm{MeV} /$ nucleon [26]. Agreement with the experimental data is obtained only if the duration time of the particle emission is large. The obtained parameter set reflects a moderately large system (Gaussian radius parameter $R_{G}=4.0 \mathrm{fm}$ ) at a 
moderate temperature $\left(T_{0}(n)=3 \mathrm{MeV}\right.$ and $\left.T_{0}(p)=5 \mathrm{MeV}\right)$ and small flow. Note, however, that the agreement between the model and the data was obtained only if the flow effect is included, i.e. within this phenomenological picture the inclusion of some flow is needed.

\section{ACKNOWLEDGMENTS}

J.H. would like to thank B. Jakobsson, R. Ghetti and the CHIC collaboration for helpful discussions, Cs.T. would like to thank D. Ardouin, B. Jakobsson and G. Gustafson for helpful discussions and M. Gyulassy and X.-N. Wang for kind hospitality. S. Pratt is acknowledged for his comments on the manuscript and for making his interferometry code available to us. This work was supported by the Training and Mobility through Research (TMR) programme of the European Community under contract ERBFMBICT950086 and by the Swedish Natural Science Research Council, by the Human Capital and Mobility (COST) programme of the EEC under grant No. CIPA - CT - 92 - 0418 (DG 12 HSMU) and by the Hungarian NSF under Grant No. OTKA-F4019 and W/01015107, by an Advanced Research Award of the Fulbright Foundation, by the Director, Office of Energy Research, Office of High Energy and Nuclear Physics, Nuclear Physics Division of the U.S. Department of Energy (Contracts No. DE-AC03-76SF00098 and No.DE-FG02-93ER40764).

\section{APPENDIX A: DETAILS OF ANALYTIC APPROXIMATIONS}

We have examined the validity of the saddle point approximation, presented in section III], both analytically and by comparing with a numerically generated particle distribution. The saddle point approximation normally yields a good approximation for large values of the expansion parameter (here $a$ ). However, for some parameter sets the saddle point approximation is not a good approximation for large $a$ values (and large momenta $p$ ). This is because for large $a$, the emission function can develop more than one maximum, and the second order expansion around one of the maxima yields a poor approximation. The range of values of $a$ and $p$ for which the saddle point approximation is valid, depends on the other parameters used.

For the case of two well separated maxima the saddle point approximation can be improved by summing up the contribution from the two maxima. Here we give the expression for the momentum distribution obtained in this approximation

$$
\begin{aligned}
N_{1}(\boldsymbol{p}) \approx & c_{g}\left(2 \pi R_{*}^{2}(\boldsymbol{p})\right)^{(3 / 2)}\left\{\exp \left(-\frac{E_{k}}{T_{* 0}}\left[\frac{1+a^{2} E_{k} R_{G}^{2} / T_{0} t_{0}^{2}}{1+a^{2} E_{k} R_{* 0}^{2} / T_{0} t_{0}^{2}}\right]\right)\right. \\
& \left.+\exp \left(-\frac{E_{k}}{T_{* 0}}\left[\frac{1+a^{2} E_{k} R_{T 0}^{2} / T_{0} t_{0}^{2}}{1+a^{2} E_{k} R_{* 0}^{2} / T_{0} t_{0}^{2}}\right]\right)\right\}
\end{aligned}
$$

An approximate expression can also be obtained for small values of the parameter $a$ by making a Taylor expansion of the emission function around $a=0$. Within this approximation we obtain (identifying the integrated result as the first terms in an expansion of the exponential function): 


$$
\begin{aligned}
N_{1}(\boldsymbol{p}) & \approx c_{g}\left(2 \pi R_{*}^{2}(\boldsymbol{p})\right)^{(3 / 2)} \\
& \times \exp \left(-\frac{E_{k}}{T_{* 0}}-\frac{15 a^{2} R_{* 0}^{4}}{4 t_{0}^{2} R_{T 0}^{2}}-\frac{a^{2} E_{k}}{T_{0} t_{0}^{2}}\left[\frac{3 R_{* 0}^{2}}{2}-\frac{5 R_{* 0}^{6}}{R_{T 0}^{2} R_{G}^{2}}\right]-\frac{a^{2} E_{k}^{2}}{T_{0}^{2} t_{0}^{2}} \frac{R_{* 0}^{8}}{R_{T 0}^{2} R_{G}^{4}}\right)
\end{aligned}
$$

In the above expressions we have used the notation

$$
R_{T 0}=R_{T}(\boldsymbol{p}=0), \quad R_{* 0}=R_{*}(\boldsymbol{p}=0) \quad \text { and } \quad T_{* 0}=T_{*}(\boldsymbol{p}=0) .
$$

Comparing these analytic approximations with numerical calculated momentum distributions we have found that for most parameter sets a good agreement can be found with either of the approximations. 


\section{REFERENCES}

[1] T. Csörgő, B. Lörstad and J. Zimányi, Phys. Lett. B338 (1994) 494, nucl-th/9408022.

[2] C. B. Chitwood et al., Phys. Rev. C 34 (1986) 858.

[3] B. Jakobsson, private communication.

[4] T. Csörgö and B. Lörstad, Phys. Rev. C54 (1996) 1390

[5] T. Csörgö and B. Lörstad, hep-ph/9612325, Heavy Ion Physics 4 (1996) 221.

[6] D.H. Boal, C.-G. Gelbke and B.K. Jennings Rev. Mod. Phys. 52 (1990) 553.

[7] W. Bauer, C.-K. Gelbke and S. Pratt, Annu. Rev. Nucl. Part. Sci. 42 (1992) 77-100.

[8] D. Ardouin, Invited talk at "International Workshop on Particle Interferometry in High Energy Heavy-Ion Reactions", ECT*, Sept. 16-27, 1996, Trento, Italy.

[9] G. F. Bertsch and S. Das Gupta, Phys. Rept. 160 (1988) 189; J. Aichelin, Phys. Rept. 202 (1991) 233-360.

[10] D. O. Handzy et al., Phys. Rev. Lett. 75 (1995) 2916.

[11] W. G. Gong et al, Phys. Rev. C43 1804 (1991);

A. Elmaani et al, Phys. Rev. C48 1864 (1993).

[12] S. Pratt, T. Csörgő and J. Zimányi, Phys. Rev. C 42 (1990) 2646.

[13] S. Chapman and U. Heinz, Phys. Lett. B340, 250 (1994)

[14] W. A. Zajc in "Particle Production in Highly Excited Matter", H. Gutbrod and J. Rafelski eds, NATO ASI series B303 (Plenum Press, 1993) p. 435.

[15] S. Chapman, P. Scotto and U. Heinz, Phys. Rev. Lett. 74 (1995) 4400; S. Chapman, P. Scotto and U. Heinz, hep-ph/9409349, Heavy Ion Physics 1 (1995) 1.

[16] J. P. Bondorf, S. I. A. Garpman and J. Zimányi, Nucl. Phys. A296 (1978) 320.

[17] W.A. Friedman and W.G, Lynch Phys. Rev. C 28 (1983) 16.

[18] S. Pratt and M.B. Tsang, Phys. Rev. C 36 (1987) 2390.

[19] W.A. Friedman Phys. Rev. Lett. 60 (1988) 2125.

[20] Yu. Sinyukov, in Proc. HHM:TE (Divonne, June 1994, Plenum Press, J. Rafelski et al., eds.)

[21] S. V. Akkelin and Yu. Sinyukov, preprint ITP - 63 - 94E.

[22] G. F. Bertsch, Nucl. Phys. A498 (1989) 173c.

[23] T. Csörgő and S. Pratt, KFKI-1991-28/A, p. 75; S. Pratt, Phys. Rev. D 33, (1986) 1314.

[24] T. Csörgő and B. Lörstad, Nucl. Phys. A590 (1995) 465c.

[25] W. G. Gong et al., Phys. Rev. C 43 (1991) 1804.

[26] M. Cronqvist et al. Phys. Lett. B317 (1993) 505.

[27] R. Ghetti et al. Nucl. Inst. Meth. A 335 (1993) 156. 